\title{
A training procedure for obtaining contrast-sensitivity functions within a single session in monkeys
}

\author{
TERRY L. DEVIETTI \\ Central Washington University, Ellensburg, Washington \\ and \\ JOHN A. D'ANDREA, DONALD J. HATCHER, and MICHAEL D. REDDIX \\ Naval Aerospace Medical Research Laboratory, Pensacola, Florida
}

\begin{abstract}
The present paper describes a training procedure that can be used to obtain contrast-sensitivity functions in a single session with monkeys (Macaca mulatta). Examples of these functions, obtained early in the testing phase of the procedure, are presented. For comparison, the functions obtained from several human volunteers performing the same procedure are also shown. The resulting methodology is versatile enough to use in a variety of sensory systems with only minor modifications.
\end{abstract}

Several recent investigations have reported a variety of biological aberrations in the eye of the monkey following exposure to microwave radiation (Kues, Hirst, Lutty, D’Anna, \& Dunkelberger, 1985; Kues et al., 1989; Kues, McLeod, D’Anna, Lutty, \& Monahan, 1988; McCally, Farrell, Bargeron, Kues, \& Hockheimer, 1986; Monahan, Kues, McLeod, D'Anna, \& Lutty, 1988). Nearly all of this research has been conducted with the anesthetized monkey. To understand the generality of the reported eye effects, however, it is necessary to evaluate the functional integrity of the visual system in the awake, alert monkey. Because the time course of the biological aberrations obtained in the above cited literature occurred across many days, it is important to achieve a functional assessment within a single experimental session so that the time course of any visual

We thank R. Upchurch, A. Thomas, C. Bates, F. Thrasher, and Petty Officers A. Ortiz and L. Buford for technical aid and the handling and care of the monkeys. Much of this work was done while T. L. DeVietti was at the Naval Aerospace Medical Research Laboratory supported by an Intergovernmental Personnel Act contract (1989-1990) and an American Society for Engineering Education award for summer faculty (1991). The views expressed in this article are those of the authors and do not reflect the official policy or position of the Department of the Navy, Department of Defense, or the U.S. Government. This research was sponsored by the Naval Medical Research and Development Command under Work Unit $62233 \mathrm{nmm} 33130.002-7001$. The animals used in this work were handled in accordance with the principles outlined in the Guide for the Care and Use of Laboratory Animals, prepared by the Committee on Care and Use of Laboratory Animals of the Institute of Laboratory Animals Resources, National Research Council, DHHS, NIH Publication No. 8523, 1985; and the Animal Welfare Act of 1966, as amended. Volunteer subjects were recruited, evaluated, and employed in accordance with the procedures specified in the Department of Defense Directive 3216.2 and Secretary of the Navy Instruction 3900.39 series. These instructions are based upon voluntary informed consent and meet or exceed the provisions of prevailing national and international guidelines. Reprint requests should be sent to Commanding Officer, Naval Aerospace Medical Research Laboratory, Attn: J. A. D'Andrea, 41 Hovey Road, Pensacola, FL 325081046 (e-mail: JDandrea@dcsuwf.dcsnod.uwf.edu). FAX (904) 452-4479. changes obtained may be followed. Contrast sensitivity was selected for the evaluation of visual function, as it yields much more information about the integrity of the visual system than do standard acuity tests (Campbell \& Green, 1965; De Valois \& Morgan, 1974; Levine \& Shefner, 1981). Several investigations have obtained contrast-sensitivity functions in nonhuman primates (e.g., Boltz, Harwerth, \& Smith, 1979; De Valois \& Morgan, 1974; Jacobs, 1977; Williams, Boothe, Kiorpes, \& Teller, 1981). However, none of the procedures of these investigations appeared readily adaptable to our needs. Blough $(1958,1966)$, Stebbins (1970), and others (Gourevitch, 1970; Rosenberger, 1970) pioneered behavioral procedures for the determination of sensory thresholds in animals that appeared more promising for our requirements. Using these techniques as a starting point, we developed a training procedure and software package to determine contrast-sensitivity functions in the monkey within a single session. The software package controls all events and allows threshold testing by any of three procedures (the method of constant stimuli, the method of limits, and the method of tracking). The specific details of the package, which also provide both itemized on-line eventby-event monitoring and session summary results and allow within-session changes in the procedure, are published elsewhere (Hatcher, DeVietti, \& D'Andrea, 1992).

The present paper describes the training procedure and presents some preliminary results of the performance of monkeys just beginning the stage of contrast threshold testing employing multiple spatial frequencies. In addition, the performance of several human volunteers in the same task is presented for comparative purposes.

\section{METHOD}

\author{
Subjects and Apparatus \\ Four experimentally naive, juvenile, male rhesus monkeys (Macaca \\ mulatta) obtained from Charles River Laboratories served as subjects.
}


These animals were approximately 18 months of age and weighed approximately $3.0 \mathrm{~kg}$ at the beginning of training. Each wore a plastic collar used in the pole-and-collar handling technique (Anderson \& Houghton, 1983). They were housed, one to a cage, in a vivarium maintained at $23^{\circ} \mathrm{C}$ with a photoperiod of 0600 (on) to 1800 (off). The animals were fed a standard primate diet (Wayne Co., $24 \%$ protein) in quantities sufficient to produce normal growth rates. This diet was supplemented daily with fresh fruit throughout training and testing. In training, the animals received a portion of their daily food ration of either $750-\mathrm{mg}$ or 1-g food pellets (Noyes Co., Formula L) as reinforcement for the behavioral task. The feeding schedule was arranged so that the monkeys were approximately $24 \mathrm{~h}$ food-deprived prior to each daily training session. In the early stages of training, when performance was poor and the smaller food pellets were used as reinforcers, the monkeys were given the balance of their daily ration in the form of standard chow immediately following the daily session with fruit given several hours later. As performance improved in latter sessions, the larger food pellets were used, and the animals received their entire ration of food in the experimental session and were given fruit immediately following the session.

The training chamber was a standard sound-attenuated metal enclosure with inside dimensions $65 \times 123 \times 122 \mathrm{~cm}$ high. It was fan ventilated and fitted with a standard food-pellet dispenser, and a speaker was mounted at floor level to provide white noise at $70 \mathrm{db}$ (A scale). A 75-W floodlight of hue similar to the CRT scope face was mounted to the ceiling slightly behind the animal to provide ambient light in the chamber. Two TV cameras (RCA, Model TC150116) were mounted above the chamber and focused through a one-way mirror $(30 \times 59 \mathrm{~cm})$ set in the top of the chamber, one to monitor the monkey and the other to monitor the CRT that displayed the contrast grate patterns. The restraining chair (Primate Products Rhesus Restrainer) was constructed of anodized aluminum and was fitted with two plastic response levers $(7 \mathrm{~cm}$ long, $1.3 \mathrm{~cm}$ in diameter) mounted vertically and in front of the animal: one to the right and one to the left. Standard microswitches were activated with each pull of a lever.

The programming of contingencies and recording of events was done with a microcomputer (Zenith Z-248) equipped with a Dascon-1 I/O expansion board, a CTM-05 multifunction countertimer (Metrobyte Corporation) for precise recording of the reaction time of the monkey, and a PCIP-SST 1- $\mathrm{Hz}$ to 5-MHz function generator (Metrobyte Corporation). The I/O board was used to regulate lever pulls so that holding of the lever by the monkey provided but a single event, and also to operate the pellet feeder. All L/O was connected to a screw terminal connector board. The function generator provided sine waves of varying frequencies and amplitudes as input to a display driver (Cushman, 1992) to generate the line and frame synchronizing signals to a raster-scan display forming the vertical grate pattern on a Tektronic 606B CRT. Voltage applied to the $z$-axis of the CRT controlled the luminance contrast value of the sine-wave grating. The scope face of the CRT was covered with an opaque, white $21 \times 28 \mathrm{~cm}$ poster board with a 3 -cm-diameter viewing port in its center to project the grating pattern, at eye level, to the monkey sitting $86 \mathrm{~cm}$ distant from the face of the CRT. The luminance of the poster board surface was equal to the luminance of the scope face ( 24 candelas per square meter). The luminance contrast values of the sine wave gratings ranged from a high of .2542 to a low of .0035 in $.06 \mathrm{log}$ steps. The $z$-axis voltages necessary to achieve these values were determined from the linear relationship between the function generator voltage and the contrast on the CRT $(r=.9999)$. Measurements of the luminance contrast on the CRT were made with a Pritchard Photometer (Model PR-1980A). The brightness of the scope face was adjusted so that the overall luminance remained constant ( 24 candelas per square meter) during all contrast presentations as well as in the absence of a contrast. Six spatial frequencies, ranging between 1.5 and $25.0 \mathrm{cpd}$ of visual angle, were available for use.

\section{Procedure}

The monkeys were gradually adapted to the handling procedure and placement in the chair. When they would rapidly pick up and eat a food pellet placed in the food tray, they were magazine trained by automatically activating the feeder on a VI 30 - to 36 -sec schedule, which delivered a food pellet into the food tray. This procedure was continued daily until the monkey remained quietly in the chair and picked up and ate the food pellets immediately following delivery.

The left lever was then attached to the chair, and a salient contrast was presented on the CRT. The monkeys were shaped to pull the left lever to produce a food pellet by reinforcing successive approximations of this response with food pellets. After several successful responses, a discrimination procedure was instituted in which a 10- to 20-sec VI schedule presented the contrast, and a left lever pull resulted in a food pellet and the disappearance of the contrast. Over sessions, the VI controlling the presentation of the contrast was increased to 30-36 sec. Responses in the absence of the contrast were never reinforced and, in addition, responses within $5 \mathrm{sec}$ of a contrast presentation resulted in a forfeiture of that presentation. In this way, responding in the absence of a contrast was never closely followed with a presentation of a contrast. During these sessions, a number of contrast values, all well above threshold, were presented randomly. In addition, the six spatial frequencies were also presented randomly to give the monkeys experience with the different frequencies at different contrast values. The monkeys continued to perform in this mode until responses to the presentation of a contrast were rapid, and very little responding occurred during the absence of a contrast.

The right lever was attached to the chair, and the monkey was shaped to respond with this lever by reinforcing approximations of a right-lever response with the presentation of a contrast. A right-lever response produced a contrast, and, with the contrast present, a left-lever response produced a food pellet and the offset of the contrast. Following several successful trials by the monkey, the right-lever responding was placed on a 10 -sec VI schedule and was gradually increased across sessions to 30-36 sec. Again, the six spatial frequencies were randomly presented in these sessions as was a greater range of contrast values, all still above threshold, to give the monkeys experience with conditions approximating threshold testing. After performance under these conditions had stabilized, several additional schedules were introduced. The duration of the contrast presentation was gradually reduced to $4 \mathrm{sec}$. With this implementation, the monkey had to respond to a contrast with a left-lever response within $4 \mathrm{sec}$, or a food pellet was forfeited as the contrast reverted to zero (i.e., no contrast). Otherwise, the contrast terminated with the left-lever response, and the reaction time was recorded. In order to enhance discrimination performance, a time-out (TO) of $5 \mathrm{sec}$ was introduced following left-lever responses in the absence of a contrast. During TO, the CRT flashed on and off at a rate of $1 \mathrm{~Hz}$, and no other schedule was in effect. Thus, responding to the left lever in the absence of a contrast not only did not yield a food pellet, it also delayed the opportunity for a contrast presentation. A "sleep-time" of $4 \mathrm{~s}$ accompanied the introduction of the TO schedule to prevent a TO from occurring following a correct left-lever response to a contrast presentation. Several animals developed the habit of responding more than once with the left lever or held the left lever during a contrast, and the addition of the TO schedule resulted in a TO after each contrast presentation as the contrast terminated immediately following the first response to the left lever. The "sleep-time" delayed the TO under these conditions. Finally, 30 catch trials were added to the schedule. Catch trials are contrast trials of zero value (i.e., no contrast), which were presented randomly during the scheduled contrast presentations. The program recorded the catch trials separately, and these data provided an index of "guessing" by the monkey. A left-lever response during a catch trial was not reinforced with food and produced a TO as well. The addition of the 30 catch trials to the contrast presentations, as well as the later introduction of threshold testing, where, by definition, some presented contrasts are below threshold and therefore not responded to, effectively increases the VI on the right lever. However, regardless of the procedural requirements, reinforcement density was maintained at 100 to 120 pellets $h$.

After the introduction of the brief contrast duration, the TO schedule, "sleep time," and the 30 catch trials, the daily sessions continued until the behavioral performance of the animals was both stable and accurate. Specifically, training continued until a steady rate of responding was achieved on the right lever, reinforcement density was relatively constant at 100-120 food pellets/session, responding to catch trials was infrequent, and the number of TO within sessions was low. When their performance met these conditions, the animals began threshold testing.

\section{Threshold Testing}

Contrasts ranging in value between .2542 and .0035 , in .06 log change units, were available for use at frequencies corresponding to $1.5,3.0$, $6.0,12.0,18.0$, and $25.0 \mathrm{cpd}$. These contrast values extend well above and below the thresholds normally obtained for this range of frequencies in the macaque (see, e.g., De Valois \& Morgan, 1974). 
Thresholds were evaluated with the tracking procedure. A contrast value above threshold was selected, and the monkey's response to this contrast dictated the contrast value of the next trial. A correct response to the contrast automatically decreased the contrast level one unit, whereas an incorrect response to the contrast increased the contrast level one unit. In this manner, the monkeys' performance oscillated about its threshold for the particular spatial frequency presented. Once approximately 10 transitions had occurred about a small range of contrast values, a different spatial frequency was presented, and the session continued until a threshold for that frequency had been obtained. Initially, one to two spatial frequencies were tested in each session, depending on the performance of the particular monkey. The number of spatial frequencies tested in a session was increased to three as performance stabilized. Over succeeding sessions, including intermittent sessions in which the method of constant stimuli was used with all contrasts presented above threshold to stabilize performance, we then attempted to obtain thresholds on five spatial frequencies (1.5, $3.0,6.0,12.0$, and $18.0 \mathrm{cpd}$ ) within a single session in all four monkeys. We did not establish thresholds for a spatial frequency of $25.0 \mathrm{cpd}$, because this frequency was difficult to synchronize consistently on the CRT, and the five remaining spatial frequencies provided sufficient information for our purposes.

Following two daily sessions after introduction of all five spatial frequencies within a session, contrast-sensitivity functions (the reciprocal of the contrast threshold) were obtained in three successive sessions. The order of the presentation of the five spatial frequencies was randomized for each day. Three human volunteers were also tested in a single session with the same spatial frequencies under conditions as close as possible to those under which the monkeys performed. The human volunteers, in strict compliance with laboratory protocol, wore gloves, mask, and protective clothing. The volunteers knelt or sat behind the monkey chair in the chamber and leaned over the chair to place their eyes in the correct position and distance from the CRT. Like the monkeys, these volunteers responded with the right lever to produce a contrast on a VI schedule and pulled the left lever to indicate the perception of a contrast. The auditory cues of the feeder provided feedback for correct performance.

\section{RESULTS AND DISCUSSION}

Within 120 sessions, all 4 monkeys had progressed, rather uniformly, from tentatively accepting food pellets placed in the food tray by the experimenter to efficient performance with both the right and left response levers in preparation for threshold testing. Occasionally, when beginning a new stage of training or adding a new requirement within a training stage-for example, the TO-the performance of 1 monkey or more would deteriorate to the extent that it became necessary to reinstate the previous contingencies to regain behavioral control. In all such cases, additional training provided a smooth transition to the new requirements of the schedule.

When threshold testing with the tracking procedure was initiated, using only one or two spatial frequencies, performance first deteriorated and then gradually improved. Relatively stable performance was obtained within 80 sessions. Early in threshold testing, when the performance of a monkey began to deteriorate about its threshold value, for example, cessation of right-lever responding and/or an excessive number of TOs, we found it useful to increase the contrast value. This allowed the monkey to experience a contrast value above threshold on the following trial, which almost always stabilized the animals' performance.

Figures 1 and 2 , respectively, show the contrastsensitivity functions of both the monkeys and the human volunteers at each of five spatial frequencies obtained in a single session. The monkey data are the second-order regression of three consecutive daily sessions initiated after the animals had experienced as many as five frequencies within a session only twice. For each monkey, the actual performance at each spatial frequency is indicated with a symbol for each the three sessions. The human data reflects only the performance in a single session. It is apparent that all subjects, both human and monkey, show the inverted U-shaped function with peak sensitivity in the 3.0- to 6.0-
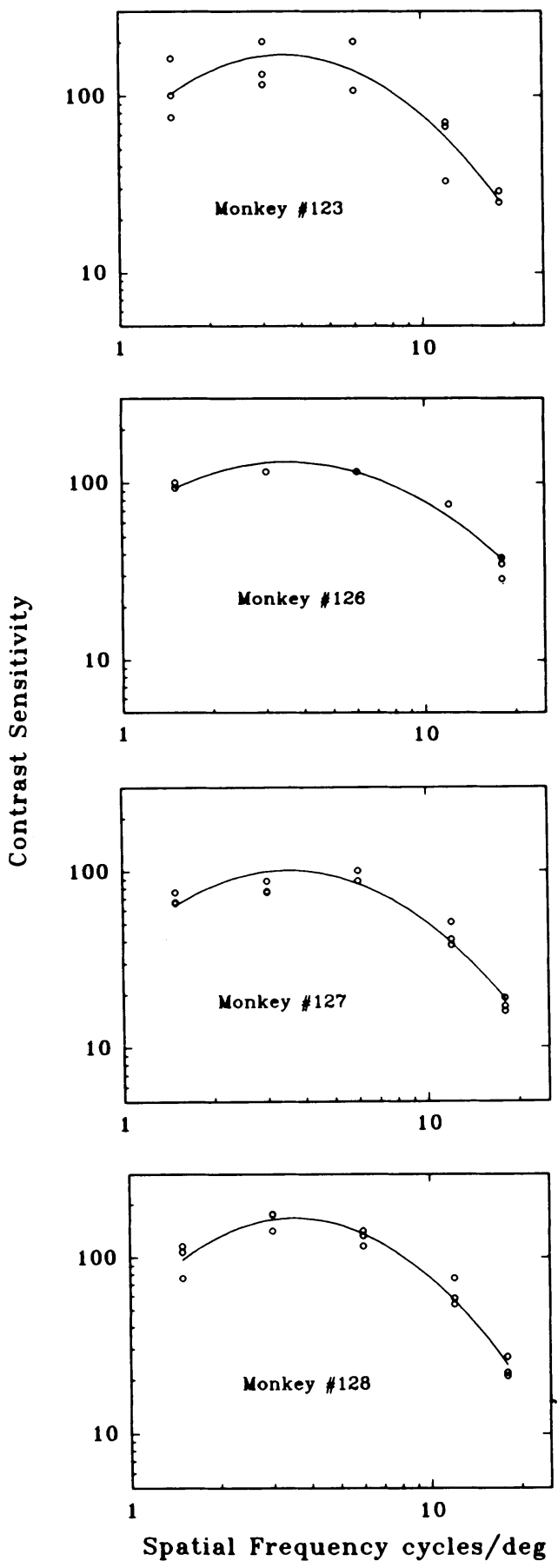

Figure 1. Monkey contrast-sensitivity functions. 


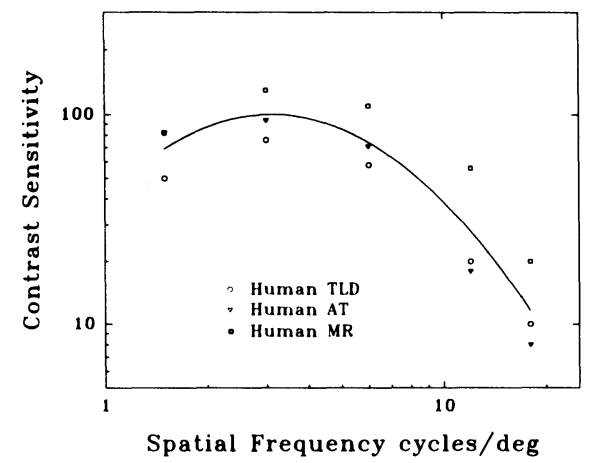

Figure 2. Human contrast-sensitivity functions.

cpd range, which is typical for the luminance conditions used and the range of spatial frequencies tested. The performance of the monkeys is very similar to contrast sensitivities reported previously for well-trained macaques using procedures requiring multiple sessions to obtain contrastsensitivity functions (DeValois \& Morgan, 1974; Williams et al., 1981), and the performance of the humans is within the $90 \%$ limits of the normative population of a commercially available diagnostic form of the test (Vistech, 1988).

Humans typically show slightly higher contrast sensitivity relative to monkeys throughout the range of spatial frequencies tested (see, e.g., DeValois \& Morgan, 1974; Williams et al., 1981). However, this does not appear to be the case in the present work. The relatively poor performance of the humans probably resulted from the novelty of the task and testing conditions and from the fact that these conditions were uncomfortable for the humans. To obtain the proper visual field, the humans had to sit or kneel behind the monkey chair, lean forward over the chair, and raise the head to place the eyes parallel to the CRT face. The maintenance of this position during testing put considerable strain on the muscles of the neck and back.

It is imperative that the intersession sensitivities of the monkeys be stable, because this performance is to serve as a baseline from which to evaluate the possible effects of low-level microwave radiation in future experiments. However, it is clear from inspection of Figure 1 that not all the monkeys meet this requirement at this early stage of testing, as is shown by the overlap, or lack thereof, of the symbols. Monkeys 126 and 127 show only slight intersession variability in their performance; there is considerable overlap of the symbols at most of the spatial frequencies. However, the performance of Monkeys 123 and 128 is too variable to provide a useful baseline, and particularly Monkey 123 will require more training to stabilize performance. Moreover, it is possible that the contrast sensitivities obtained in Monkeys 126 and 127, even though apparently quite stable in this early stage, may increase with further training - an effect that has been noted by other investigators using different procedures (e.g., DeValois \& Morgan, 1974; Jacobs, 1977).

In any event, the present procedure appears promising as a technique with which to generate contrast-sensitivity functions in the monkey within a single, relatively short, test session.

\section{REFERENCES}

Anderson, J. H., \& Houghton, P. (1983). The pole and collar system: A technique for handling and training nonhuman primates. Lab Animal, $12,47-49$.

BLough, D. S. (1958). A method for obtaining psychophysical thresholds from the pigeon. Journal of the Experimental Analysis of Behavior, 1 , 31-43.

BLough, D. S. (1966). The study of animal sensory processes by operant methods. In W. K. Honig (Ed.), Operant behavior: Areas of research and application (pp. 345-379). New York: Appleton-CenturyCrofts.

Boltz, R. L., Harwerth, R. S., \& SMith, E. L. (1979). Orientation anisotropy of visual stimuli in rhesus monkey: A behavioral study. Science, 205, 511-513.

Campbell, F., \& Green, D. (1965). Optical and retinal factors affecting visual resolution. Journal of Physiology, 181, 576-593.

Cushman, W. B. (1992). A low-cost spatial contrast sensitivity display driver. Behavior Research Methods, Instruments, \& Computers, 24, 461-463.

De Valois, R. L., \& Morgan, H. (1974). Psychophysical studies of monkey vision: III. Spatial luminance contrast sensitivity tests of macaque and human observers. Vision Research, 14, 75-81.

Gourevitch, G. (1970). Detectability of tones in quiet and in noise by rats and monkeys. In W. C. Stebbins (Ed.), Animal psychophysics (pp. 67-97). New York: Plenum.

Hatcher, D. J., DeVietti, T. L., \& D'Andrea, J. A. (1992). Computer software and hardware to determine contrast sensitivity using three methods: Tracking, limits, and constant stimuli. (Tech. Memorandum No. 92-4). Pensacola, FL: Naval Aerospace Medical Research Laboratory.

JACOBS, G. H. (1977). Visual capacities of the owl monkey (Aotus trivirgatus): II. Spatial contrast sensitivity. Vision Research, 17, 821-825.

Kues, H. A., Hirst, L. W., Lutty, G. A., D'Anna, S. A., \& DunkelBERGER, G. R. (1985). Effects of 2.45-GHz microwaves on primate corneal endothelium. Bioelectromagnetics, 6, 177-188.

Kues, H. A., Mcleod, D. S., D'Anna, S. A., Lutty, G. A., GamBRILL, C., \& ELSON, E. C. (1989, June). Ocular changes following exposure to high-peak pulsed $1.25 \mathrm{GHz}$ microwaves. Paper presented at the meeting of the Bioelectromagnetics Society, Tucson, AZ.

Kues, H. A., McLeod, D. S., D'Anna, S. A., Lutty, G. A., \& MonaHAN, J. C. (1988, June). Histological evaluation of microwave-induced vascular leakage in the iris. Paper presented at the meeting of the Bioelectromagnetics Society, Stamford, CT.

Levine, M. W., \& ShefNer, J. M. (1981). Fundamentals of sensation and perception. Reading, MA: Addison-Wesley.

McCally, R. L., Farrell, R. A., Bargeron, C. B., Kues, H. A., \& HoCKHEIMER, B. F. (1986). Nonionizing radiation damage in the eye. Johns Hopkins APL Technical Digest, 7, 73-91.

Monahan, J. C., Kues, H. A., McLeod, D. S., D'Anna, S. A., \& LUTTY, G. A. (1988, June). Lowering of microwave exposure threshold for induction of primate ocular effects by timolol maleate. Paper presented at the meeting of the Bioelectromagnetics Society, Stamford, CT.

ROSENBERGER, P. B. (1970). Response-adjusting stimulus intensity. In W. C. Stebbins, (Ed.), Animal psychophysics (pp. 161-184). New York: Plenum.

Stebrins, W. C. (1970). Studies of hearing and hearing loss in the monkey. In W. C. Stebbins (Ed.), Animal psychophysics (pp. 41-66). New York: Plenum.

Vistech Consultants, InC. (1988). Instruction manual. Dayton, OH: Author.

Williams, R. A., Boothe, R. G., Kiorpes, L., \& Teller, D. A. (1981). Oblique effects in normally reared monkeys (Macaca nemestrina): Meridional variations in contrast sensitivity measured with operant techniques. Vision Research, 21, 1253-1266.

(Manuscript received December 16, 1992.) 